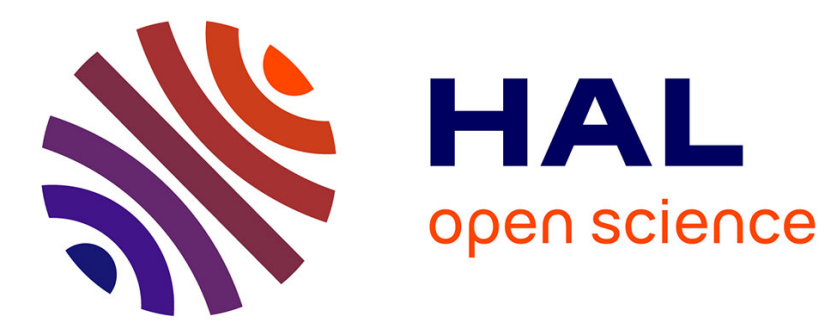

\title{
Josef Dobrovský et le criticisme tchèque de la fin du XIXe siècle
}

\author{
Catherine Servant
}

\section{To cite this version:}

Catherine Servant. Josef Dobrovský et le criticisme tchèque de la fin du XIXe siècle. Revue des études slaves, 2002, 74 (2-3), pp.411-418. 10.3406/slave.2002.6810 . hal-01291991

\section{HAL Id: hal-01291991 \\ https://hal-inalco.archives-ouvertes.fr/hal-01291991}

Submitted on 25 Mar 2016

HAL is a multi-disciplinary open access archive for the deposit and dissemination of scientific research documents, whether they are published or not. The documents may come from teaching and research institutions in France or abroad, or from public or private research centers.
L'archive ouverte pluridisciplinaire HAL, est destinée au dépôt et à la diffusion de documents scientifiques de niveau recherche, publiés ou non, émanant des établissements d'enseignement et de recherche français ou étrangers, des laboratoires publics ou privés.

\section{(이) $\$$}

Distributed under a Creative Commons Attribution - NonCommercial - NoDerivatives $\mid 4.0$ 


\section{Josef Dobrovský et le criticisme tchèque de la fin du XIXe siècle}

\section{Madame Catherine Servant}

Abstract
Josef Dobrovsky and czech criticism at the end of the 19th century.

In the 1880s, the thought of Josef Dobrovsky (1753-1829) has become a main reference for the linguistic, historiographie and ethical discussions of the 'realistic' scientists united around Tomás Garrigue Masaryk (1850-1937). This acknowledgement of Dobrovsky's scientific and symbolic leadership, along with a somewhat reductive interprétation of his héritage, marks an attempt to introduce in the national thought of the 19th-century Czech lands a line of criticism linking the enlightenment period to the fin de siècle radically critical orientations.

\section{Citer ce document / Cite this document :}

Servant Catherine. Josef Dobrovský et le criticisme tchèque de la fin du XIXe siècle. In: Revue des études slaves, tome 74, fascicule 2-3, 2002. pp. 411-418.

doi : $10.3406 /$ slave.2002.6810

http://www.persee.fr/doc/slave_0080-2557_2002_num_74_2_6810

Document généré le 08/09/2015 


\section{JOSEF DOBROVSKÝ \\ ET LE CRITICISME TCHÈQUE DE LA FIN DU XIXe SIÈCLE}

PAR

\section{CATHERINE SERVANT}

Dans les années 1880, le nom de Josef Dobrovský (1753-1829), fondateur de la tradition philologique tchèque et figure associée aux commencements de la Renaissance nationale, traverse les discussions linguistiques, historiographiques et proprement éthiques des universitaires et chercheurs réalistes réunis autour de Tomáš Garrigue Masaryk (1850-1937) et de sa revue critique Athenaeum (fondée en 1883). Le resurgissement du « patriarche » s'avère particulièrement décisif au moment où les réalistes ouvrent une nouvelle fois, en 1886, un débat qui forme l'une des grandes trames de l'histoire littéraire et scientifique, mais aussi «identitaire » des Tchèques au long du XIX ${ }^{e}$ siècle : le débat des (faux) Manuscrits de Zelená Hora et Dvůr Králové.

Autorité philologique et morale, premier adversaire du Manuscrit de Zelená Hora dans les années 1820, incarnation d'un esprit des Lumières finalement étouffé par le romantisme des éveilleurs nationaux réunis sous la bannière de Josef Jungmann (1773-1847), Dobrovský opère, à plusieurs titres, en référence fondatrice, tant scientifique que proprement symbolique, dans l'émergence du réalisme scientifique des années 1880 , à l'heure où la science tchèque définit et autonomise son champ d'action au sein de l'organisme national. Or, au terme du cheminement archétypal représenté par l'aventure des Manuscrits - de la mystification à la démystification, de la foi à la critique... -, revenir à Dobrovský signifie aussi réintégrer, dans l'histoire de la pensée nationale tchèque, une ligne de permanence critique à la faveur de laquelle se trouvent reliées les Lumières et cette phase de criticisme total qui caractérise la fin du $\mathrm{XIX}^{\mathrm{e}}$ siècle tchèque.

Après leur prétendue « découverte » dans la société patriotique tchèque de la fin des années 1810 - du vivant de Dobrovský —, les Manuscrits de Dvůr Králové et Zelená Hora, promptement intégrés au patrimoine littéraire et historique de la nation tchèque alors en pleine renaissance, s'installent à demeure dans le domaine public des objets de culte les plus intouchables : "médiévaux », fragmentaires, recelant de magnifiques poèmes lyriques, épiques et lyrico-épiques rédigés dans une langue tenue pour du vieux tchèque, les 
Manuscrits vont occuper une place capitale dans l'ordre des mythes et stéréotypes constitutifs du discours national tout au long du XIXe siècle. Leur puissance symbolique hors du commun, les impulsions et orientations qu'ils donnent à la création littéraire et artistique tchèque, leurs incidences, des plus fécondes aux plus fâcheuses, sur la production scientifique, leur rôle essentiel dans l'historicisme à la base des revendications émancipatrices et politiques tchèques... tout cela constitue autant d'obstacles à leur destitution. Dans la seconde moitié du XIX siècle, lors même que leur authenticité est déjà fortement sujette à caution, toute menace pesant sur les vieux poèmes continue de provoquer de violentes réactions de défensive. Près de soixante-dix ans sépareront donc l'apparition des Manuscrits de leur révocation publique.

Les hommes de plume les plus probablement impliqués dans la falsification - V. Hanka (1791-1861), J. Linda (1789 ou 1792-1834) et V. A. Svoboda (1791-1849) — se sont pourtant heurtés, peu après l'avènement des Manuscrits, à l'opposition vigoureuse de Dobrovský - et c'est d'abord en cette qualité de « grand devancier » qu'il sera remis à l'honneur dans les années 1880. Dès 1818, le philologue émet en effet, en privé, des doutes sur l'authenticité du Jugement de Libuše (premier titre du Manuscrit de Zelená Hora), soupçonnant Linda, Hanka et même leur maître à penser Jungmann d'en être les auteurs et les «fabricants». En 1824, au comble de l'agacement devant la fortune du Manuscrit (édité et étudié en Pologne ${ }^{1}$, céjà réédité en tchèque, déjà traduit en allemand...), Dobrovský publie un article incendiaire intitulé Une tromperie littéraire $^{2}$ où il met principalement en évidence, chez ses cadets, la motivation patriotique qui se substitue de plus en plus dangereusement à un minimum d'honnêteté scientifique - chose inacceptable pour lui, savant d'une intégrité et d'un rationalisme venus d'un autre âge ; il invective l'imposteur « ... qui a osé tromper non point le monde cultivé, mais quelques fervents et crédules patriotes, au plus haut point réjouis de pouvoir exhiber, dans leur langue, un document des plus anciens ${ }^{3} »$. Peu après, en réplique à Dobrovský, V. A. Svoboda engage une querelle d'une étonnante bassesse, censée disqualifier le savant aussi bien que 1'homme et le patriote 4 . Svoboda n'avance que de maigres arguments scientifiques, comptant avant tout sur les attaques personnelles pour réduire au silence cet adversaire de taille : il accuse « Herr D. » d'être « un homme hypercritique, [...] aveuglé par l'orgueil vaniteux de son autorité et de son infaillibilité critique, un renégat doué d'assez de sentiment non patriotique [unpatriotisch] pour considérer comme falsification, supercherie ou chimère et rejeter purement et simplement tout ce qui pourrait attester la culture ancienne de sa nation, à la honte de cette dernière, dont il fait partie, qui l'aime et l'honore ». Svoboda le traite encore d'homme « atteint d'idées fixes», ne luttant qu'« à coups de

1. Dans le vol. I de l'anthologie Prawda ruska, éditée par J. B. Rakowiecki (Warszawa, 1920), dont Dobrovský donne en 1824 une critique (anonyme) dans les Jahrbücher der Literatur (Wien).

2. J. Dobrovský, «Literarischer Betrug », p. 152-153, in Literární a prozodická bohemika [Études littéraires et prosodiques tchèques], Prague, Academia, 1974 (repris de la revue viennoise Archiv für Geographie, Historie, Statistik, Staats- und Kriegskunst, 1824).

3. Ibid., p. 152.

4. V. A. Svoboda, «Libusa als Gesetzgeberin » [Libuše rendant la justice], p. 157164, in : J. Dobrovský, Literárni a prozodická bohemika, op. cit. (repris de Archiv für Geographie..., 1824). 
formules violentes, inhumaines $»^{5}$, etc. Très touché, Dobrovský se défend dans une réponse polémique ${ }^{6}$ non dénuée d'ironie ( "Comme il va rire dans sa barbe, l'auteur futé du poème envoyé au Musée, le Jugement de Libuše, en voyant avec quelle flamme on défend à présent son galimatias ${ }^{7} \gg$ ), considérant avec force lucidité les malversations patriotiques qui sous-tendent la défense du Manuscrit et résumant déjà les « représailles » suscitées par son rejet :

[Les fervents patriotes littéraires tchèques] ont trouvé très inconvenant mon jugement défavorable parce qu'il les perturbait dans leur joie d'avoir fait cette inestimable découverte. À présent, ils méprisent le jugement formulé, après mûre réflexion, par un homme qui, certes, n'est pas né parmi eux, mais a été élevé parmi eux, un homme qui a toujours eu à cœur de voir la gloire nationale tchèque dans les choses littéraires, et ils qualifient ce jugement de non patriotique. [...] En distinguant le vrai du faux, en menace-t-on pour autant la gloire nationale tchèque ${ }^{8}$ ?

Pendant des décennies, nombreux seront ceux qui se heurteront au même mur. Dès cette époque, qui exprime ses doutes se rend passible d'exclusion de la société nationale pour «non-patriotisme » — et cet élément est à prendre en compte tout autant (sinon plus) que l'insuffisance réelle des connaissances sur le Moyen Âge à l'orée du XIX $X^{\mathrm{e}}$ siècle.

Après 1829, année où disparaît Dobrovský, autorité scientifique devenue bien gênante, et où une autre figure de proue de la Renaissance nationale, l'historien František Palacký (1798-1876), fait son entrée sur la scène publique de la controverse en prenant le parti des Manuscrits, l'acceptation sine qua non des deux monuments poétiques devient une attitude parfaitement « recommandée » dans la société patriotique tchèque. Suit une ère d'apogée et de foi quasiment inconditionnelle. Seuls quelques audacieux, dont le philologue Jernej Kopitar (1780-1844) $)^{9}$ vers 1840 , oseront adopter une position contraire. Il faut attendre les années 1880 pour qu'unc jcune équipe scientifique issue de l'Université tchèque nouvellement fondée entreprenne, sous la conduite de T. G. Masaryk, une démonstration méthodique de la supercherie. Dès lors, il ne s'agit plus seulement d'engager une solide enquête pour lever le voile, définitivement, sur « un secret de polichinelle ${ }^{10} \gg$; le temps est surtout venu, à travers des initiatives d'ordres intellectuel, culturel, politique, d'instaurer un regard neuf, plus objectif sur l'existence nationale tchèque - ses fixations et blocages, ses survivances romantiques, son attachement démesuré à l'histoire... L'intervention de 1886 provoque naturellement un vaste débat opposant les membres d'Athenaeum philologues, historiens, historiens littéraires... - à tout un bloc patriotico-journalistique et dégénérant en une crise politique grave qui ébranle pour un temps

5. Ibid., p. 158-159, passim.

6. J. Dobrovský, « Vorlaüfige Antwort auf des Herrn W. S. Ausfälle » [Réponse provisoire aux attaques de Monsieur W. S. (V. Svoboda)], p. 154-156, in Literární a prozodická bohemika... (repris de Archiv für Geographie..., 1824).

7. Ibid., p. 154.

8. Ibid.

9. «Ce "Méphisto" de Vienne, élève de Dobrovský, qui en savait très long - et qui, le premier, fut tenu chez nous pour un ennemi des Manuscrits à la solde du gouvernement viennois » (Václav Flajšhans, Jak to vlastně bylo? [Que s'est-il vraiment passé ?], Praha, Historický klub, 1932).

10. Formule de Masaryk (Athenaeum, t. 3, 1885-1886, p. 165). 
la vie publique tchèque ; une crise où le politique intervient plus manifestement que jamais - élément omniprésent dans un débat qui n'a pour ainsi dire jamais revêtu un caractère véritablement scientifique et littéraire. À la fin du siècle, la question est réglée pour la majorité de la communauté scientifique tchèque, qui tient les Manuscrits pour des créations contemporaines de leur « découverte ».

Dans les années 1880, la nouvelle controverse des Manuscrits semble sauver Dobrovský de la longue «traversée du désert » dans laquelle l'ont entraîné, quelque soixante ans auparavant, ses conflits avec les «Jungmannovci » - sur l'authenticité du Jugement de Libuše, mais aussi sur d'autres questions philologiques, prosodiques, historiographiques... Comme tend à le prouver le petit nombre d'articles et ouvrages monographiques consacrés au savant de sa disparition à la fin du siècle ${ }^{11}$, la fortune de Dobrovský en pays Tchèques demeure longtemps méciiocre et indécise, bien inférieure à ce que laissaient présager sa longue carrière et ses nombreux écrits. Parmi les chefs d'accusation les plus tenaces, on reproche à Dobrovský de n'avoir été qu'un analyste insensible et obtus, de ne pas avoir ajouté foi aux chances de survie de la langue et de la nation tchèques (son célèbre « ich zweifle » de 1791), d'avoir refusé d'abandonner l'allemand au profit du tchèque pour la rédaction de ses travaux (le Slawisierender Deutscher que Jungmann voyait en lui), de s'être montré individualiste et indifférent à la cause nationale... On lui oppose presque mécaniquement les écrivains de la génération de Jungmann - et au premier chef, Jungmann lui-même, auteur d'une ouvre déterminante et patriote patenté, grand homme ayant ressuscité la nation de ses cendres, mentor des plus jeunes... La représentation de ce couple antagoniste est courante dans l'opinion publique, surgissant même au cœur des discours de cette «politique par la culture » qui caractérise la vie publique tchèque à partir des années 1860 . Un exemple parmi d'autres de déclaration officielle sur les deux hommes : en 1873, pour le centenaire de la naissance de Jungmann, le chef de file du Parti vieux-tchèque František Ladislav Rieger (1818-1903) célèbre la grandeur des éveilleurs « ... qui eurent l'audace de ramener à la vie cette nation dont Dobrovský ne croyait déjà plus qu'elle fût viable » avant de rappeler que, par bonheur, «Jungmann vint, qui rendit sa langue à la nation $[\ldots] »^{12}$, etc.

Ainsi que le résume Arne Novák, Dobrovský a donc longuement pâti du complexe de valeurs romantiques qu'il avait combattu - culte de l'autorité, surestimation des légendes, fantaisie et sentiment comme moteurs de la reconstruction du passé, absence d'esprit critique dans l'appréciation de l'existence nationale ${ }^{13} \ldots$ La dépréciation de Dobrovský, qui se perpétue globalement jusqu'aux années 1880 , ne fait en somme que prolonger le jugement émis sur lui, de son vivant, par ses adversaires. Même le (vain) antagonisme qui oppose le savant scrupuleux à l'esprit de mystification et à la «peste de falsification » (selon une expression de Kopitar) surclassant peu à peu le souci de la vérité scientifique, est une «situation-type» prise en compte par ses

11. Il s'agit pour l'essentiel des deux monographies de Fr. Palacký et de Vincenc Brandl (1834-1901), philologue et historien morave, parues respectivement en 1833 et 1883 - leurs auteurs partageant paradoxalement, outre l'admiration pour Dobrovský, une même foi en l'authenticité des Manuscrits.

12. Cité par H. Traub dans Fr. L. Rieger, Praha, SVU Mánes, 1922, p. 356.

13. Cf. Arne Novák, Josef Dobrovský, Praha, SVU Mánes, 1928, p. 81. 
détracteurs contemporains, comme le montre dès 1824 cet argument édifiant avancé par Svoboda :

Nous serions heureux si nous comptions, nous aussi, parmi nous un Chatterton, et nous le prierions, sans mettre d'accent particulier sur la précision historique de ses travaux, de composer beaucoup d'œuvres de cette sorte. [...] Car de toute notre âme, nous sommes convaincus qu'un Chatterton génial est plus à même de profiter à notre culture que des hommes qui dépeuplent les siècles par leur critique exagérée ${ }^{14}$.

Surprenants propos où l'apologie de l'auteur des Poésies de Thomas Rowley, tout en rendant 1' « hypercritique » Dobrovský nuisible au progrès de la Renaissance nationale, inscrit sans ambages le «cas » des Manuscrits dans un univers de mystification ordinaire.

À la fin du XIXe siècle, la balance se met à pencher du côté de Dobrovský - et ce changement d'image, loin de se limiter à la controverse des Manuscrits, est bientôt répercuté et développé par l'histoire et surtout l'histoire littéraire. En premier lieu, les scientifiques rassemblés en 1886 autour de la destitution des Manuscrits se placent d'emblée sous le « patronage » de Dobrovský parce que la langue des Manuscrits est au centre de la démonstration ; Dobrovský sert d'appui aux analyses linguistiques présentées, entre autres, par le principal protagoniste en ce domaine ${ }^{15}$, le philologue Jan Gebauer (1838-1907). Or, si Dobrovský fait rapidement figure de savant modèle, c'est dans une acception beaucoup plus large : tout d'abord, ses méthodes et assises scientifiques - souci des faits avérés, rationalisme, voire même recours à des procédés inductifs proches des sciences exactes... - lui permettent d'être perçu comme une sorte de « pré-réaliste scientifique » et tissent un lien indéfectible entre les Lumières (dont Dobrovský est vu comme l'incarnation la plus aboutie en pays Tchèques) et l'équipe constituée autour de T. G. Masaryk, marquée par des conceptions et méthodes nouvelles — non sans lien avec le « positivisme ambiant ». Ensuite, dans la véritable leçon de déontologie scientifique que les réalistes dispensent alors à leurs opposants ${ }^{16}$, Dobrovský fait véritablement office d'appui et d'exemple : champion de la vérité, ne se départant jamais de son intégrité intellectuelle ni de son regard critique, rejetant impitoyablement les erreurs, supputations et inventions, esprit iconoclaste luttant pour la liberté de pensée et contre tout fanatisme, religieux ou patriotique... ce « vrai chevalier de l'esprit ${ }^{17}$ », ainsi que le nomme Masaryk dans sa Question tchèque de 1895, semble réunir toutes les qualités ambitionnées pas les jeunes savants. Il correspond en particulier à leur haute idée de la responsabilité individuelle des penseurs, écrivains, savants, et surtout, de la charge morale dont doit être investie toute pratique scientifique ${ }^{18}$.

14. Svoboda, op. cit., p. 164.

15. C'est un article de Jan Gebauer (paru dans Athenaeum, t. 3, 1885-86, p. 152-164) qui déclenche les hostilités en 1886.

16. Cf. C. Servant, Critique et nation: la naissance de la critique dans les lettres tchèques (1860-1890), Montpellier, Presses de l'université Paul-Valéry, 2000, chap. « Le réalisme scientifique : vers une "critique totale"? », p. 185-251.

17. T. G. Masaryk, Česká otázka [la Question tchèque], Praha, Čin, 1948, p. 42 [1 re éd. 1895$]$.

18. On trouve notamment cette analyse dans le vaste chapitre consacré par Jan Jakubec à « Dobrovský éveilleur » - cf. « Dobrovský buditel », in Literatura česká deva- 
À travers leur analyse de la première querelle des Manuscrits en 1824 comme d'un grand «moment éthico-scientifique », les réalistes savent également apprécier chez Dobrovský une conception moderne - fondamentalement anti-romantique - de l'appartenance nationale, en vertu de laquelle préférer le vrai au faux ne signifie pas être moins patriote, tout au contraire. Jan Jakubec (1862-1936), historien littéraire issu du réalisme scientifique, rappelle que si Dobrovský doutait de la viabilité du tchèque, refusait d'écrire en tchèque ou se montrait implacable dès lors qu'il s'agissait de dénoncer un faux, son sentiment national n'en était pas moins puissant ( Dobrovský se sentait toujours sincèrement tchèque et le déclarait partout 19 »); dans sa Question tchèque, où il œuvre vigoureusement à la réhabilitation de Dobrovský - ses qualités « positives », sa « vertu littéraire », sa « droiture critique ${ }^{20} \ldots$ - - Masaryk met particulièrement en valeur le doute chez Dobrovský, rappelant que même ses adversaires doutaient (fût-ce en privé) des destinées de la nation, que ce doute n'a pas empêché Dobrovský de travailler — « ... telle une mère près du berceau de son enfant chétif ${ }^{21} »$-, enfin, que «... son scepticisme a beaucoup plus aidé notre renaissance et notre conscience slave que les certitudes de tous les Hanka et autres patriotes du même esprit ${ }^{22}$ ».

Depuis la formidable campagne lancée par Athenaeum contre les Manuscrits en 1886 jusqu'au premier volume de la Littérature tchèque du $X_{X}{ }^{e} s_{i e ̀ c l e}{ }^{23}$ (1902) - monument de la nouvelle école d'histoire littéraire (qualifiée de positiviste) née du creuset réaliste -, en passant par la Question tchèque de Masaryk (1895), le rapport des réalistes à Dobrovský laisse transparaître, outre une indéniable identification avec le savant, comme une solidarité à distance avec les combats qu'il a menés. Cette parenté est à double tranchant : d'une part, si la polémique de 1824 apparaît comme un débat caractéristique du passage scientifique, mais aussi idéologique et moral, des Lumières au romantisme, assimiler les prises de position de Dobrovský à celles des réalistes (à plus de soixante années de distance) signifie faire le constat d'une emprise durable conservée par le romantisme sur la vie artistique, intellectuelle et politique de la nation tchèque tout au long du XIX $\mathrm{X}^{\mathrm{e}}$ siècle. Ne cessant d'analyser cette «mécanique romantico-patriotique » tenace qu'ils s'efforcent d'enrayer - tout particulièrement dans le domaine historiographique - , les réalistes désignent la toutepuissance d'un État de fiction dans lequel s'éternise l'ensemble de la vie nationale, responsable d'une stagnation dont les sciences fournissent un indice majeur.

D'un autre côté, la présence d'un Dobrovský au cœur de la Renaissance nationale permet aux réalistes de tracer dans l'histoire du XIX' tchèque une ligne de permanence critique dont Dobrovský est tenu pour le grand initiateur. « $\mathrm{Ce}$

tenáctého století [la Littérature tchèque du XIXe siècle], t. I : Od Josefa Dobrovského $k$ Jungmannově škole básnické [De J. Dobrovský à l'école poétique de Jungmann], œuvre collective de J. Hanư̌, J. Jakubec, J. Máchal, E. Smetánka, J. Vlček, Praha, J. Laichter, 1902, p. 120200.

19. Jakubec, ibid., p. 193.

20. Cf. Masaryk, Ceská otázka, p. 41-42, passim.

21. Ibid., p. 42.

22. Ibid., p. 37.

23. Literatura česká devatenáctého století, t. I. 
fut une véritable bénédiction qu'au berceau de notre renaissance, il y ait eu un esprit aussi critique et honnête pour guider la jeune génération », écrit Masaryk $^{24}$. De là, celui que Masaryk appelle encore "notre père spirituel » peut devenir la figure avant-courrière d'un criticisme tchèque «s'épanouissant " finalement, à la fin du siècle, dans les sciences et avant peu — avec la «critique moderne » des années 1890 -, en littérature. $L$ ' « hypercriticisme » est devenu la plus haute qualité de l'homme ${ }^{25}$ et de son œuvre - et même un étendard sous lequel placer le travail du temps présent.

Cette vision n'échappe pas à un schématisme certain, comme le signale l'inversion des valeurs qui s'opère alors entre Dobrovský et Jungmann - cette bipolarité devenue traditionnelle tournant à présent, de manière assez brutale, aux dépens de Jungmann. Sévèrement stigmatisé26, ce dernier semble alors « sacrifié27 » à Dobrovský, dont le profil sort nettement embelli de ce retournement : on a tendance à encenser sa perspicacité scientifique en oubliant qu'il n'a pas toujours été infaillible - un autre historien littéraire, Josef Hanuš, lui pardonne ainsi de n'avoir pas su percer l'inauthenticité du Manuscrit de Dvůr Králové28, précisant que Dobrovský se montrait tout de même de plus en plus dubitatif sur la question ${ }^{29}$. En outre, le débat symbolique de 1824 fait de Dobrovský le premier d'une série de victimes du mensonge et du faux. L'historien Jaroslav Goll (1846-1929), autre acteur majeur de la campagne antiManuscrits de 1886, déclare par exemple en 1886: «... je ne crains pas de voir apparaitre mon nom, tôt ou tard, sur la liste des proscrits. En haut de cette liste, on trouve les noms d'hommes de bien, ceux de Dobrovský et Kopitar, auxquels jamais je n'oserais moi-même joindre le mien ${ }^{30}$. " Ainsi que le note Oldřich Králík, c'est donc un Dobrovský principalement considéré dans la dernière décennie de sa vie, vieillard hérö̈que assailli et éclipsé par ses élèves ingrats, qui fait son apparition dans les années $1880^{31}$.

«Après la grande victoire de la critique scientifique sur les Manuscrits, Josef Dobrovský revient en triomphe, tel un Cid mort, dans le monde scientifique tchèque, devenant un objet de vénération qui donne naissance à une nouvelle légende, pour le moins surprenante chez des ennemis déclarés des légen-

24. Česká otázka, p. 42.

25. À propos de Dobrovský, Masaryk insiste encore (ibid.) sur sa «... vigilance critique, et surtout, son aversion envers tous les comportements faux et immatures qui apparaissaient déjà dans notre camp, dans l'intérêt présumé de la patrie [...] ».

26. Josef Hanuš accuse notamment Jungmann d'avoir contribué à ternir l'image de Dobrovský après sa mort - cf. « První období sporův o pravost památek podvržených » [la Première Période de la querelle sur l'authenticité des monuments falsifiés], in Literatura česká devatenáctého století, p. 894.

27. Formule de A. Novák, op. cit., p. 5.

28. En effet, tout en s'exprimant avec prudence sur le Manuscrit de Dvůr Králové, Dobrovský l'a jugé authentique et en a intégré des extraits, dès 1818 , à la réédition de son ouvrage Geschichte der böhmischen Sprache und ältern Literatur (1 ${ }^{\mathrm{re}}$ éd. 1792).

29. Cf. Hanus, op. cit., p. 870.

30. «Z Gollova "Historického rozboru básní RK" » [Extrait de l'Analyse historique de poèmes du Man. de D.K., de J. Goll], p. 331, in : Jaroslav Marek, Jaroslav Goll, Praha, Melantrich, 1991 [ $1^{\text {re }}$ ed. 1886].

31. Cf. Oldřich Králík, « Josef Dobrovský a Gelasius Dobner » [J. D. et G. D.], p. $95-$ 98, in Osvobozená slova [Mots en liberté], Praha, Torst, 1995 [1 re éd. 1953]. 
des 32 », écrit A. Novák en 1928. Au XXe siècle, tandis que les appréciations plus nuancées de l'histoire littéraire ne placent plus forcément Dobrovský et Jungmann en «concurrence » ou en opposition, on voit apparaître l'idée que Dobrovský a peut-être été l'instrument, à la fin du XIXe siècle, d'une relecture utile de la Renaissance nationale. Ce Dobrovský idéalisé, écrit Novák, occulte un temps «... le Dobrovský réel [qui] occupe si peu les spécialistes qu'ils n'ont toujours pas publié ses œuvres complètes ni achevé de publier sa correspondance 33 ».

Or, force est de remarquer que la «lecture » spécifique de l'histoire tchèque proposée par Masaryk dans sa Question tchèque, ainsi que les analyses de la nouvelle histoire littéraire, dotent aussi Dobrovský d'un successeur quelque peu inattendu : le poète, journaliste et penseur politique Karel Havlíček Borovský (1821-1856). Jakubec nomme Dobrovský «notre Havlíček du XVIII' siècle ${ }^{34}$ » tandis que Masaryk présente Havlíček comme le continuateur de l'homme des Lumières : «Après Dobrovský, de nouveau un esprit véritablement critique ; et il fallait la critique pour que la fausseté de notre vie intérieure soit éliminée ${ }^{35}$. » De prime abord, cette filiation paraît bien surprenante : qu'y at-il de commun entre l'abbé Dobrovský, figure érudite du XVIII' siècle, philologue pointu au-dessus de la mêlée patriotique, et un journaliste dont l'action politique a été capitale aux alentours de 1848 , devenu un symbole héroíque de l'opposition anti-autrichienne... ? Outre l'affirmation de la personnalité et du caractère - notion devenue capitale en cette fin de siècle - , un criticisme irréductible sur des assises rationalistes? L'accent mis sur cette filiation nous donne de comprendre que pour le « réalisme scientifique » émergeant dans les années 1880 , restituer la continuité d'un «esprit de critique » dans l'histoire tchèque du siècle qui s'achève constitue peut-être le moyen le plus sûr d'entériner la rupture avec la Renaissance nationale.

\section{SUMMARY}

\section{JOSEF DOBROVSKÝ AND CZECH CRITICISM \\ AT THE END OF THE 19TH CENTURY}

In the 1880s, the thought of Josef Dobrovský (1753-1829) has become a main reference for the linguistic, historiographic and ethical discussions of the 'realistic' scientists united around Tomás Garrigue Masaryk (1850-1937). This acknowledgement of Dobrovský's scientific and symbolic leadership, along with a somewhat reductive interpretation of his heritage, marks an attempt to introduce in the national thought of the 19th-century Czech lands a line of criticism linking the enlightenment period to the fin de siecle radically critical orientations.

(Institut national des langues et civilisations orientales, Paris)

32. Novák, op. cit., p. 6.

33. Ibid., p. 2.

34. Jakubec, op. cit., p. 125.

35. Masaryk, Ćeská otázka, p. 105. 